\title{
Transoral Incisionless fundoplication for reflux after peroral endoscopic myotomy: a crucial addition to our arsenal
}

다 (i)

\author{
Authors \\ Amy Tyberg, Anthony Choi, Monica Gaidhane, Michel Kahaleh
}

Institution

Rutgers Robert Wood Johnson Medical School, Rutgers, The State University of New Jersey, New Brunswick, NJ USA

submitted 5.12.2017

accepted after revision 5.2.2018

Bibliography

DOI https://doi.org/10.1055/a-0584-6802 |

Endoscopy International Open 2018; 06: E549-E552

(c) Georg Thieme Verlag KG Stuttgart · New York

ISSN 2364-3722

Corresponding author

Michel Kahaleh, MD, AGAF, FACG, FASGE, Professor of Medicine, Clinical Director of Gastroenterology, Chief of Endoscopy, Director Pancreas Program, Department of Medicine, Rutgers Robert Wood Johnson Medical School, Rutgers, The State University of New Jersey, Robert Wood Johnson University Hospital, 1 RWJ Place, MEB 464, New Brunswick, NJ 08901

Fax: 732-235-5537

mkahaleh@gmail.com

\section{ABSTRACT}

Introduction Increased esophageal acid exposure is seen in a large percentage of patients with achalasia who undergo peroral endoscopic myotomy (POEM). Endoscopic trans- oral fundoplication (TIF) is a novel endoscopic technique for the management of patients with chronic gastroesophageal reflux (GERD). We present the first case series evaluating the role of TIF post-POEM.

Methods Consecutive patients 18 years or older from our academic institution who underwent a POEM procedure and subsequently underwent TIF for symptomatic reflux or regurgitation between December 2014 and June 2017 were included. The primary outcome was discontinuation of proton-pump inhibitor (PPI) use and healing of esophagitis (when initially present) on post-procedure esophagogastroduodenoscopy (EGD). Technical success was defined as successful completion of the endoscopic fundoplication. Adverse events (AEs) were recorded for all patients.

Results Five patients were included (60\% male, average age $55 \pm 14$ years). Technical success was achieved in $100 \%$ of patients. Discontinuation of PPI use was achieved in 5/5 patients (100\%). Three patients had esophagitis pre-procedure and all were noted to have resolution of inflammation on post-procedure EGD. No adverse events were noted. Mean follow-up time was 27 months (range 5-34 months). Conclusion TIF post-POEM appears feasible, safe, and efficacious in improving symptoms and esophagitis, decreasing long-term risks of acid exposure, and decreasing risks of long-term PPI use in patients post-POEM in this small cohort of patients. Larger studies are needed to confirm these initial findings.

\section{Introduction}

Peroral endoscopic myotomy (POEM) has emerged as a safe and efficacious therapeutic option for patients with achalasia with efficacy rates upwards of $90 \%$ [1]. However, with successful decrease in lower esophageal sphincter (LES) pressure to relieve symptoms of dysphagia comes the reciprocal consequence of increased acid exposure into the esophagus often requiring long-term proton-pump inhibitor (PPI) use [1-5]. Given the known association between long-standing gastroesophageal reflux (GERD) and the development of Barrett's esophagus (BE) [6] and esophageal adenocarcinoma [7], as well as the in- creasing adverse health effects of long-term PPI use [8, 9], such high incidences of post-procedural esophageal acid exposure have significant potential consequences.

Endoscopic transoral fundoplication (TIF) is a novel therapeutic endoscopic technique for the management of patients with chronic GERD [10]. The feasibility of performing TIF after POEM has previously been described in a single video case presentation [11]. We present the first case series evaluating the role of TIF for management of post-POEM GERD. 

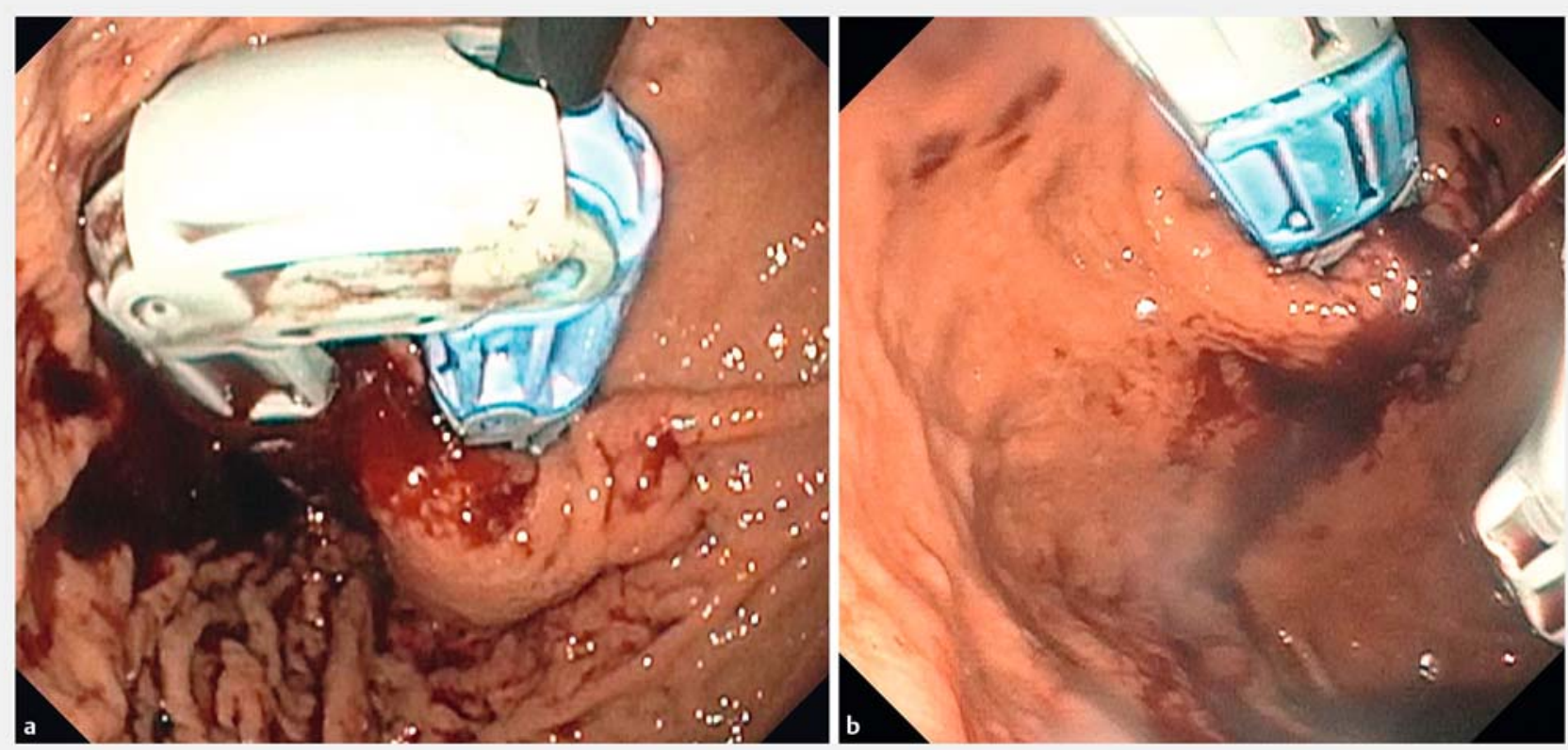

- Fig. 1 a, b Esophyx device creating the wrap.

\section{Methods}

\section{Study overview}

Consecutive patients 18 years or older who underwent a POEM procedure and subsequently underwent a TIF at our institution between December 2014 and June 2017 were included in a prospective registry. All included patients had reflux symptoms post-POEM. All included patients were offered TIF versus longterm PPI therapy or surgical fundoplication and opted for TIF. Patient demographic information, clinical and procedural data, and follow-up data were collected for all patients. The institutional review board approved the prospective registry (ClinicalTrials.gov Identifier: NCT02162589).

\section{Definitions}

The primary outcome was discontinuation of proton-pump inhibitor (PPI) use and healing of esophagitis (when initially present) on post-procedure esophagogastroduodenoscopy (EGD). Technical success was defined as successful completion of the endoscopic fundoplication. Adverse events (AEs) were recorded for all patients.

\section{Procedural technique}

All procedures were performed under general anesthesia by endoscopists (AT, MK) with expertise in performing TIF. Periprocedural antibiotics were given in all cases. A flexible endoscope (GIFH 190; Olympus; Center Valley, PA, United States) was used to evaluate the esophagus and assess for a hiatal hernia before the procedure. The Esophyx $Z$ device (Endogastric Solutions; Redmond, WA, United States) was then introduced transorally over a flexible endoscope (GIF-XP190, Olympus) into the stomach. The endoscope was retroflexed and the plastic jaw was advanced fully into the stomach under direct visua-

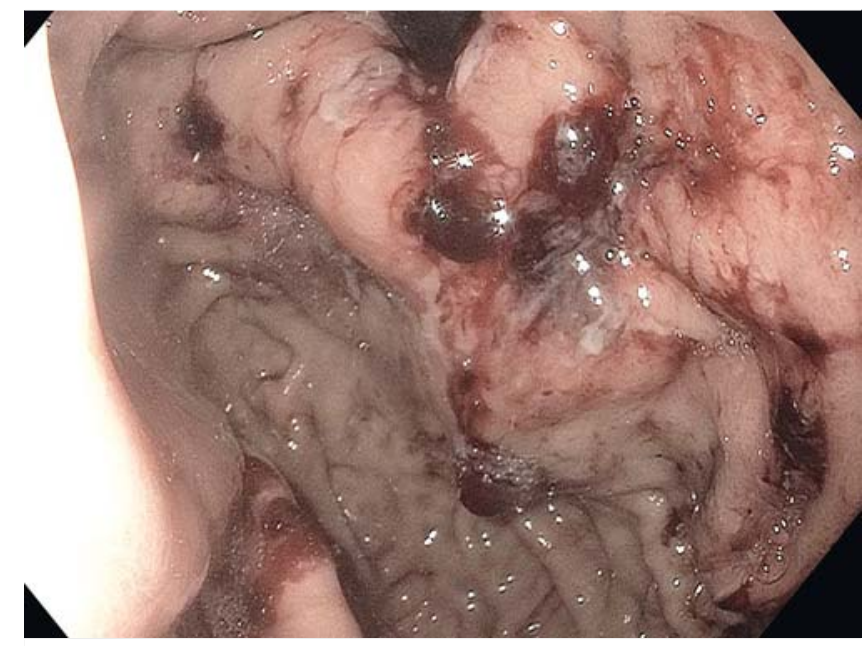

- Fig. 2 Successful 270 degree fundoplication.

lization. The endoscope was retracted back into the device and the plastic jaw was closed. Subsequently, the endoscope was re-advanced into the gastric lumen and retroflexed to gain visualization of the device. The plastic jaw was rotated to the 11 o'clock position and oriented to just proximal to the lip of gastric tissue distal to the gastroesophageal junction ( $\mathbf{F i g . 1 a ) . ~}$ The helical retractor was inserted into the gastric tissue, coiled and retracted to secure the tissue within the device ( $\triangleright$ Fig. 1 b). The device was then rotated counter-clockwise creating the wrap, and the plastic jaw was closed and locked in place. An invaginator was activated to avoid involvement of the diaphragm. Polypropylene fasteners were then deployed through the apposed esophageal and gastric walls. The invaginator was then disengaged and the tissue released. The process was then 
- Table 1 TIF post-POEM patient data.

\begin{tabular}{|c|c|c|c|c|c|c|c|}
\hline & Age, years & Gender & Indication for TIF & $\begin{array}{l}\text { On PPI } \\
\text { pre-TIF }\end{array}$ & $\begin{array}{l}\text { Esophagitis on } \\
\text { EGD pre-TIF }\end{array}$ & $\begin{array}{l}\text { Off PPI } \\
\text { post-TIF }\end{array}$ & $\begin{array}{l}\text { Healed esophagitis } \\
\text { post-TIF }\end{array}$ \\
\hline Patient 1 & 39 & $\mathrm{~F}$ & + pH study & $\mathrm{Y}$ & Y, Class B & $\mathrm{Y}$ & $\mathrm{Y}$ \\
\hline Patient 2 & 70 & $\mathrm{~F}$ & Regurgitation & Y & $\mathrm{N}$ & Y & $\mathrm{n} / \mathrm{a}$ \\
\hline Patient 3 & 51 & $M$ & + pH study & Y & Y, Class D & Y & Y \\
\hline Patient 4 & 44 & $\mathrm{M}$ & + pH study & Y & $\mathrm{N}$ & Y & $\mathrm{n} / \mathrm{a}$ \\
\hline Patient 5 & 69 & $M$ & + pH study & Y & $\mathrm{Y}$, Class B & Y & $\mathrm{Y}$ \\
\hline
\end{tabular}

repeated until a 270 degree fundoplication was achieved ( $\triangleright$ Fig.2). The device was then removed through the orophar$y n x$, and a flexible endoscope was used to examine the fundoplication. A total of 20 fasteners were used in each procedure. Post-procedure, all patients were admitted to the hospital for at least 1 night for observation.

\section{Outcome measurements}

Patients were continued on PPI for 6 weeks post-TIF procedure after which they were tapered off as tolerated. All patients underwent follow-up endoscopy post-TIF at 3 months post-procedure. All patients were evaluated post-procedure in follow-up via an office visit and/or phone encounter.

\section{Results}

Five patients were included $(60 \%$ male, average age $55 \pm$ 14 years). Two patients (40\%) were status-post posterior approach POEM, two patients (40\%) were post anterior approach POEM, and one patient (20\%) underwent anterior followed by posterior POEM. All patients were on PPIs pre-TIF with either complete improvement or some improvement without complete relief. All patients underwent $\mathrm{pH}$ testing post-POEM and pre-TIF. Three patients had positive DeMeester scores on $\mathrm{pH}$ study with esophagitis on EGD, two categorized as class B and one categorized as class $\mathrm{D}$; one patient had a positive $\mathrm{pH}$ study without esophagitis, and one patient had regurgitation symptoms ( $\triangleright$ Table 1$)$.

\section{Procedural data}

Technical success was achieved in $100 \%$ of patients. The average amount of time between POEM and TIF was 13.5 months (range 4-27 months). All patients spent at least 1 night in the hospital post-TIF; one patient spent 2 nights.

\section{Primary and secondary outcomes}

Discontinuation of PPI use was achieved in 5/5 patients (100\%). Post-procedure EGD was performed in all patients 3 months post-procedure. All patients with esophagitis pre-procedure were noted to have resolution of inflammation on post-procedure EGD. Four out of five patients had their PPIs stopped 6 weeks after TIF; one patient remained on PPIs for 1 year post-procedure due to persistent symptoms despite resolution
- Table 2 TIF post-POEM results.

\begin{tabular}{|l|l|}
\hline Technical success & $100 \%(n=5)$ \\
\hline Off of PPI & $100 \%(n=5)$ \\
\hline Healing of esophagitis & $100 \%(n=2)$ \\
\hline Adverse events & 0 \\
\hline Mean follow-up time & 27 months (range 5-34 months) \\
\hline $\begin{array}{l}\text { POEM, peroral endoscopic myotomy; PPI, proton-pump inhibitor; TIF, trans- } \\
\text { oral fundoplication. }\end{array}$
\end{tabular}

of esophagitis on EGD but has been off PPIs since. No patients had $\mathrm{pH}$ testing post-TIF. Average follow-up time was 27 months (range 5-34 months). There were no adverse events ( $\triangleright$ Table 2).

\section{Discussion}

Patients with achalasia who undergo POEM have high rates of esophageal acid exposure putting them at risk for development of $\mathrm{BE}$ and esophageal adenocarcinoma [6,7]. Routine post-procedure $\mathrm{pH}$ testing and EGD evaluation have demonstrated rates of abnormal acid exposure and esophagitis in the range 28 $58 \%$ and $17-31 \%$, respectively in published series [ $1-5$ ]. Endoscopic fundoplication (TIF) is an exciting new treatment option for patients with GERD. The procedure involves creation of a 270-330 degree full-thickness esophagogastric fundoplication via an over-the-scope device [9]. In randomized control trials, this technique has shown superiority over high-dose medical therapy and/or a sham procedure in relieving GERD symptoms with efficacy rates comparable to surgical Nissen fundoplication $[12,13]$. However, the procedure is indicated only for patients with a hiatal hernia less than $2 \mathrm{~cm}$, somewhat limiting its widespread generalizability. Patients who are status-post POEM and suffering from increased acid exposure represent an ideal population to benefit from TIF. These patients do not have a hiatal hernia as a contributing cause of their reflux, and due to their underlying achalasia, they would in theory benefit from a 270 - 330 degree wrap compared to a 360 degree surgical wrap to prevent recurrent post-procedure dysphagia. Additionally, 
they are potentially at increased surgical risk due to scarring from the myotomy.

To date, only one video case report has demonstrated the feasibility and safety of performing TIF post-POEM [11]. Our study represents the first case series of 5 patients who successfully underwent TIF after POEM procedure. We showed $100 \%$ technical success and no adverse events, confirming the procedure to be safe and feasible. Additionally, $100 \%$ of patients were able to discontinue their PPIs and all patients with esophagitis had resolution of their inflammation on repeat EGD, confirming that the procedure was also efficacious.

In patients with achalasia who undergo Heller myotomy (HM), a surgical fundoplication is typically performed concurrently. Although rates of esophageal reflux are lower post-HM compared to POEM with this technique, a recent meta-analysis showed that over $10 \%$ of patients are still affected and at a cost of a mild decrease in dysphagia efficacy [14]. Combining POEM with staged TIF allows for selective treatment of only those patients who require treatment for acid exposure. Additionally, separating the myotomy from the fundoplication may allow for post-procedure healing from each intervention, again increasing efficacy.

Recently, concerns have emerged over the association between many adverse health risks and long-term PPI use. These include kidney disease, dementia, bone disease, micronutrient deficiencies, infections such as pneumonia and clostridium difficile, and most recently gastrointestinal malignancy, specifically gastric cancer $[8,9]$. While these health risks are mainly felt to be associative risks and a cause and effect relationship is yet to be clearly elucidated, cessation of PPI use after POEM is especially important to avoid potential complications related to these medications.

The main limitation of this study is the small number of patients. Additionally, while esophagitis improved and patients were able to come off their PPIs, objective $\mathrm{pH}$ testing post-TIF was not performed. And lastly, TIF was performed from a range of 4-27 months post-POEM in our study; the optimal timing post-POEM is yet to be elucidated.

In conclusion, TIF post-POEM appears feasible, safe, and efficacious in improving symptoms and esophagitis, decreasing long-term risks of acid exposure, and decreasing risks of longterm PPI use in patients post-POEM in this small cohort of patients. Patients post-POEM with symptomatic reflux should be offered TIF as a therapeutic option. Larger prospective studies are needed to confirm these initial findings.

\section{Competing interests}

Michel Kahaleh MD has received grant support from Boston Scientific, Fujinon, EMcision, Xlumena Inc., W.L. Gore, MaunaKea, Apollo Endosurgery, Cook Endoscopy, ASPIRE Bariatrics, GI Dynamics, NinePoint Medical, Merit Medical, Olympus and MI Tech. He is a consultant for Boston Scientific, Xlumena Inc., Concordia Laboratories Inc, ABBvie, and MaunaKea Tech. All other authors have no conflicts of interest to report. Dr. Amy Tyberg is a consultant for EndoGastric solutions.
References

[1] Nabi Z, Ramchandani M, Chavan R et al. Per-oral endoscopic myotomy for achalasia cardia: outcomes in over 400 consecutive patients. Endosc Int Open 2017; 5: E331-E339

[2] Repici A, Fuccio L, Maselli R et al. Post-poem reflux a systematic review and meta-analysis. Paper presented at Digestive Disease Week; May 5-9, 2017; Chicago, IL. Presentation number 1221

[3] Wu QN, Zhang X, Li Q et al. Outcomes of peroral endoscopic myotomy for treatment of esophageal achalasia with a median follow-up of 4 years. Paper presented at Digestive Disease Week; May 5-9, 2017; Chicago, IL. Presentation number 218

[4] Kumbhari V, Familiari P, Bjerregaard NC et al. Gastroesophageal reflux after peroral endoscopic myotomy: a multicenter case-control study. Endoscopy 2017; 49: 634-642

[5] Guo H, Yang H, Zhang X et al. Long-term outcomes of peroral endoscopic myotomy for patients with achalasia: a retrospective singlecenter study. Dis Esophagus 2017; 30: 1-6

[6] Johansson J, Hakansson HO, Mellblom L et al. Prevalence of precancerous and other metaplasia in the distal oesophagus and gastro-oesophageal junction. Scand J Gastroenterol 2005; 40: 893-902

[7] Hvid-Jensen F, Pedersen L, Drewes AM et al. Incidence of adenocarcinoma among patients with Barrett's esophagus. NEJM 2011; 365: $1375-1383$

[8] Freedberg DE, Kim LS, Yang YX. The risks and benefits of long-term use of proton pump inhibitors: expert review and best practice advice from the American Gastroenterological Association. Gastroenterology 2017; 152: $706-715$

[9] Cheung KS, Chan EW, Wong AYS et al. Long-term proton pump inhibitors and risk of gastric cancer development after treatment for Helicobacter pylori: a population-based study. Gut 2018; 67: 28 - 35

[10] Bell RC, Cadiere GB. Transoral rotational esophagogastric fundoplication: technical, anatomical, and safety considerations. Surg Endosc 2011; 25: 2387-2399

[11] Kumta NA, Kedia P, Sethi A et al. Transoral incisionless fundoplication for treatment of refractory GERD after peroral endoscopic myotomy. Gastrointest Endosc 2015; 81: 224-225

[12] Trad K, Fox M, Simoni G et al. Transoral fundoplication offers durable symptom control for chronic GERD: 3-year report from the TEMPO randomized trial with a crossover arm. Surg Endosc 2017; 31: 2498 2508

[13] Hunter JG, Kahrilas PJ, Bell RC et al. Efficacy of transoral fundoplication vs omeprazole for treatment of regurgitation in a randomized controlled trial. Gastroenterology 2015; 148: 324-333

[14] Schlottmann F, Luckett D], Fine J et al. Laparoscopic Heller myotomy versus peroral endoscopic myotomy (POEM) for achalasia: A systematic review and meta-analysis. Ann Surg 2018; 267: 451 - 460

\section{CORRECTION}

Tyberg A, Choi A, Gaidhane M et al. Transoral Incisionless fundoplication for reflux after peroral endoscopic myotomy: a crucial addition to our arsenal

Endoscopy International Open 2018; 06: E549-E552. DOI: 10.1055/a-0584-6802

In the above mentioned article was a mistake in the title. Correct is: Transoral Incisionless fundoplication for reflux after peroral endoscopic myotomy: a crucial addition to our arsenal 